\title{
Effects of semantic incompatibility upon deductive reasoning
}

\author{
LAWRENCE T. FRASE, BELL TELEPHONE LABORATORIES, \\ Murray Hill, N. J. 07974
}

Two hundred forty-one college Ss judged the validity of 48 syllogistic arguments. The arguments varied in terms of the connotative incompatibility of the words combined in the conclusions, and whether the conclusion was particular or universal. Both factors significantly influenced reasoning errors, but they did not interact. Results suggest that qualifiers affect cognitive interactions within assertions of a verbal hierarchy (having small but significant effects), while quantifiers affect interactions among different assertions (resulting in gross judgmental effects).

Deductive reasoning is an important component of intelligence. In certain respects the reasoning process follows predictably from research conducted on verbal learning processes. When Ss judge the validity of syllogistic arguments, their judgments are a function of the temporal sequence in which the subject, middle and predicate terms occur (Frase, 1968), the semantic compatibility of those terms, and whether the conclusion is a universal or particular assertion (Frase, 1966b).

In a previous study (Frase, 1966b), words were rated by Ss on semantic differential evaluative scales, and then combined in subject-predicate pairs to produce syllogistic conclusions of varying connotative incompatibility. Results indicated a curvilinear relationship between incompatibility and reasoning errors, with most errors made on conclusions of moderate incompatibility. Since only $25 \%$ of the syllogisms used were valid, compatible connotative meanings would seem to facilitate deductive reasoning rather than merely increasing the acceptability of a conclusion. In addition, fewest errors were made on universal conclusions. The present study attempted to replicate these results and to control possible confounding effects of the middle term (which does not occur in the conclusion) by using middle terms which lack semantic content.

Method

The Ss were 241 introductory psychology students at the University of Massachusetts who had no formal training in logic.

The same syllogisms were used as in the previous study (Frase, 1966b), except that the middle term was replaced in all syllogisms with the letter $X$. The incompatibility levels of the syllogisms used in the present study were thus based upon the ratings of words by the 64 Ss from the previous study. There were eight universal and eight particular syllogisms, each occurring with a low, moderate, or highly incompatible subject-predicate assertion in the conclusion. The universal and particular syllogisms were identical except that the conclusion began with "some" for particular syllogisms, and "all" for universal syllogisms. These two classes of syllogisms also violated the same logical rules if they were invalid.

The syllogisms were mimeographed on $81 / 2 \times 11$ sheets of paper, and randomized in eight blocks of six experimental conditions for each $S$. The S's task was to judge the validity of each of the 48 syllogisms. Ss were told to make their judgments only on the basis of whether the conclusion followed from the premises and not upon their feelings about the truth or falsity of the conclusion.

Table 1

Percentage of Correct Judgments for All Conditions

\begin{tabular}{lrrrrrrr} 
& \multicolumn{3}{c}{ Universal } & \multicolumn{3}{c}{ Particular } \\
\hline & Low & Med & High & & Low & Med & High \\
\cline { 2 - 8 } M & 71.38 & 70.25 & 69.13 & & 47.63 & 48.13 & 46.13 \\
SD & 1.27 & 1.34 & 1.44 & 1.63 & 1.65 & 1.70 \\
\hline
\end{tabular}

The experiment was administered as a test in a large auditorium during one class period. Each $S$ saw all 48 experimental syllogisms, hence the design was 2 (universal-particular conclusion) by 3 (low-medium-high incompatibility), with repeated measures on both factors. Number of correct responses was the dependent variable.

\section{Results and Discussion}

The results reported in Table 1 confirm those of the previous study. The main effect of incompatibility was significant $(F=$ 3.11 , df $=2 / 480, p<.025$ ), with fewest correct judgments occurring when the semantic content of the words used in the conclusion was highly incompatible. Both low and medium levels differed from high $(\mathrm{p}<.05)$, but did not differ from each other, hence the U-shaped relationship between semantic incompatibility and reasoning errors suggested by the previous study (Frase, $1966 \mathrm{~b})$ was not confirmed. The interaction between in compatibility and quantifier was not significant $(F=.5)$. Considering both studies, it seems most likely that there is a monotonic increase in reasoning errors as semantic incompatibility increases.

More errors were made on particular conclusions $(F=450.32$, df $=1 / 240, p<.001)$. Although the universal and particular conclusions used in the present study violated similar logical rules, Ss tended to accept an invalid conclusion if the conclusion was stated in the form "Some mothers are evil," rather than "All mothers are evil." This effect is partially the result of changes in the credibility of a conclusion (disregarding the premises upon which it is based) when the quantifier is changed. It has been shown (Frase, 1966a) that variation in quantifiers correlates .91 with belief in the conclusion. Errors in reasoning could thus result from interactions within Ss' belief hierarchy, which are under the control of quantitative terms (cf. Fishbein, 1961). The results of the present study thus confirm the earlier research in that qualitative terms (with semantic content) have small but significant effects upon the ease of reasoning, while quantitative terms ("all" or "some") produce relatively gross changes in judgments by arousing Ss' belief structure. In the present study, about $27 \%$ of the variance in judgments was accounted for by the quantifiers (estimate $\omega^{2}$ ), while only $1 \%$ was related to the incompatibility factor.

In terms of a mediation model, errors in judgment are contingent upon the incompatibilities of the words associated, but even more critically, they are contingent upon the number of relations which are asserted within the hierarchy to which they refer. Osgood (1963) has suggested that qualifiers have additive effects in cognitive interactions, while quantifiers interact in a multiplicative fashion. The present study offers confirming evidence for this assertion. Qualitative terms thus control cognitive interactions within assertions, while quantitative terms control interactions among a large class of assertions.

The lack of interaction between semantic incompatibility and quantifying terms further suggests that these two sets of terms operate in a relatively independent manner.

\section{REFERENCES}

FISHBEIN, M. An investigation of the relationship between beliefs about an object and the attitude toward that object. Tech. Rep. No. 6, Dept. Psychol., Univer. Calif., March, 1961.

FRASE, L. T. Belief, incongruity, and syllogistic reasoning. Psychol. Rep., $1966 a, 18,982$.

FRASE; L. T. Validity judgments of syllogisms in relation to two sets of terms. J. educ. Psychol, 1966b, 57, 239-245.

FRASE, L. T. Associative factors in syllogistic reasoning. J. exp. Psychol., 1968, 76, 407-412.

OSGOOD, C. E. On understanding and creating sentences. Amer. Psychologist, 1968, 18, 735-751. 